\title{
Construção de um instrumento de coleta de dados de enfermagem em ginecologia*
}

\author{
Construction of an instrument to collect nursing data in gynecology \\ Construcción de un instrumento de colecta de datos de enfermería \\ ginecología
}

\author{
Lenir Honório Soares ${ }^{1}$, Francisca das Graças Salazar Pinelli², \\ Ana Cristina Freitas de Vilhena Abrão ${ }^{3}$
}

\begin{abstract}
RESUMO: Trata-se de pesquisa Survery descritivo exploratório. Teve como objetivo a construção de um instrumento de coleta de dados, visando a implantação da sistematização da assistência de enfermagem em uma clínica de cirurgias ginecológicas. O instrumento foi elaborado com base no Modelo Bifocal da Prática Clínica de Carpenito, construído segundo os Padrões Funcionais de Saúde descritos por Gordon. Buscou-se na elaboração do instrumento uma forma de organizar e registrar um maior número de informações sobre as condições gerais e especiais de saúde das pacientes. Foi aplicado em 100 mulheres internadas em uma clinica ginecológica de um Hospital Público Estadual de Ensino do Município de São Paulo, no período de maio a setembro de 2002. Os resultados mostraram que o instrumento construído foi adequado, pois, possibilitou a identificação de 48 diagnósticos de enfermagem. Destaca-se que foram identificados oito diagnósticos numa freqüência de 70,0\% a 100\%, sendo que: "Risco para infecção, conforto alterado, déficit de conhecimento e medo"ocorreram em 100\% e, Ansiedade, Comportamento para elevar o nível de saúde, Distúrbio no padrão do sono e Integridade da pele prejudicada, numa freqüência de $96,0 \%$ a $70,0 \%$. Os demais ocorreram numa freqüência de $31,0 \%$ a $64,0 \%$.
\end{abstract}

Descritores: Coleta de dados; Diagnóstico de enfermagem; Enfermagem obstétrica

ABSTRACT: this is a descriptive exploratory survey. The purpose was to construct an instrument to collect data aiming implantation to systematize nursing assistance in a gynecologic clinic. The instrument was elaborated based on the Bifocal Clinical Practice Model developed by Carpenito constructed according to the Functional Health Patterns described by Gordon. When elaborating the instrument, a method to organize and register a greater number of information on general and special conditions of the patient's health. It was applied in 100 women hospitalized in a gynecologic clinic of a Public Teaching Hospital in the city of São Paulo, from May to September 2002 . The results showed that the constructed instrument was adequate because it made possible to identify 48 nursing diagnosis. Eight diagnoses were identified in a frequency of $70 \%$ to $100 \%$, being "Risk of Infection, Altered Comfort, Deficit of knowledge and Fear" occurred in $100 \%$ and "Anxiety, Behavior to increase Health Status, Disorder in the sleep pattern and Impaired Skin Integrity was observed in a frequency of $96.0 \%$ to $70.0 \%$. The others variables occurred in a frequency of $31.0 \%$ to $64.0 \%$

Descriptors: Data collection; Nursing diagnosis; Obstetrical nursing

RESUMEN: Este estudio es una pesquisa descriptiva exploratoria y tuvo como objetivo la construcción de un instrumento de colecta de datos, almejando la sistematización de la asistencia de enfermería en una clínica de cirugías ginecológicas. El instrumento fue elaborado con base en el Modelo Bifocal de la Práctica de Carpenito, construido según los Padrones Funcionales de la Salud descriptos por Gordon. Se buscó en la elaboración del instrumento una forma de organizaar y registrar el mayor número de informaciones sobre las condiciones generales y especiales de la salud de los pacientes. Fue aplicado en 100 mujeres internadas en un clínica ginecológica de un Hospital Público Estadual de Enseñanza del Municipio de São Paulo, en el periodo de mayo a septiembre de 2002. Los resultados mostraron que el instrumento construido fue adecuado una vez que posibilitó la identificación de 48 diagnósticos de enfermería. Fueron identificados ocho diagnóstios en una frecuencia de $70.0 \%$ a $100 \%$, siendo que "Riesgo para infección, Conforto alterado, déficit de conocimiento y miedo" ocorrieron en $100 \%$ y, "Ansiedad, Comportamiento para elevar el nivel de la salud, disturbio en el padrón del sueño e integridad de la piel perjudicada", ocurrieron en $96 \%$ a $70.0 \%$. Las demás variables ocurrieron en una frecuencia de $31.0 \%$ a $64.0 \%$

Descriptores: Recolección de dados; Diagnóstico de enfermería; Enfermería obstétrica

\footnotetext{
Parte extraída da Dissertação de Mestrado em Enfermagem na UNIFESP.

1 Mestre em Enfermagem pela UNIFESP.

2 Professora Doutora da Disciplina Enfermagem Obstétrica do Departamento de Enfermagem da UNIFESP.

3 Professora Doutora da Disciplina Enfermagem Obstétrica do Departamento de Enfermagem da UNIFESP.
} 


\section{INTRODUÇÃO}

A construção de um instrumento de coleta de dados em enfermagem, visa documentar as informações de forma objetiva, científica e compreensiva, permitindo a identificação de diagnósticos de enfermagem e, conseqüentemente, a determinação de ações de enfermagem gerando uma assistência de melhor qualidade.

A coleta de dados pode ser definida como um processo permanente, que começa quando você encontra o paciente pela primeira vez e continua em cada encontro, até que ele receba alta ${ }^{(1)}$.

O levantamento de dados é o primeiro componente do processo e consiste na coleta de dados deliberada e sistematizada, com objetivo de delinear o perfil do estado da saúde do paciente, família e comunidade ${ }^{(2)}$.

Estudos no sentido de identificação de diagnósticos de enfermagem através da prática assistencial, vêm sendo desenvolvidos desde a década de 20. Em 1950, Mcmanus, classificou o termo" diagnóstico" como uma atividade de enfermagem, descreveu as funções do enfermeiro para a identificação dos problemas, e os fatores relacionados, levando à ações de enfermagem na busca de soluções de problemas ${ }^{(3)}$.

Em 1973, um grupo de enfermeiras norte americanas realizaram a $1^{\mathrm{a}}$ Conferência Nacional sobre a classificação de diagnósticos de enfermagem, sendo apresentados e validados 34 diagnósticos de enfermagem nesta conferência ${ }^{(3-4)}$.

O elemento chave para um diagnóstico de enfermagem preciso é a coleta e análise de dados que constitui o fundamento no qual se baseia a identificação de necessidades, respostas e problemas individuais ${ }^{(5)}$.

É nessa fase que se faz uma investigação sistemática e deliberada de dados para a determinação do estado de saúde atual e passado do paciente, seu estado funcional e avaliação do seu padrão de enfrentamento de problemas presentes e passados ${ }^{(6)}$.

Um roteiro sistematizado de levantamento de dados sobre a situação de saúde do ser humano, torna possível a identificação dos seus problemas ${ }^{(7)}$.

O histórico de enfermagem é um guia sistematizado para o levantamento de dados com objetivo de se conhecer os problemas de enfermagem vivenciados pelos pacientes, para que a assistência seja direcionada ao atendimento de suas necessidades em sua globalidade, e que o atendimento contemple sua individualidade, com suas crenças e valores específicos ${ }^{(8)}$.

$\mathrm{Na}$ área de enfermagem obstétrica observa-se a realização de pesquisas com o objetivo de construção de instrumentos de coleta de dados, para a identificação de diagnósticos e conseqüientemente, intervenções de enfermagem.
Abrão ${ }^{(9)}$ construiu e validou um modelo especifico para assistência de enfermagem à puérpera e seu filho no período puerperal e de aleitamento materno.

Em 1999, foi elaborado um instrumento de levantamento de dados para assistência à mulher durante o acompanhamento pré-natal em consultas realizadas por enfermeiros e alunos de graduação e pós-graduação em enfermagem ${ }^{(10)}$.

Estudo similar, foi desenvolvido na área de sexualidade da mulher ${ }^{(11)}$.

Em enfermagem ginecológica, encontramos um estudo desenvolvido ${ }^{(12)}$, cuja finalidade foi a construção de um instrumento de coleta de dados que resultou na identificação de diagnósticos de enfermagem em consultas de enfermagem ginecológica, realizadas com mulheres em Unidade Básica de Saúde.

Desta forma, o interesse em desenvolver esta pesquisa, surgiu da observação clínica, no exercício da profissão de enfermeira, atuando na área ginecológica de uma Instituição Pública Estadual, que resultou na constatação da necessidade de utilização de um instrumento de sistematização da assistência de enfermagem.

Neste sentido, o estudo teve como objetivo, construir um instrumento de coleta de dados de enfermagem em ginecologia, visando a implantação da Sistematização da Assistência de Enfermagem (SAE) no referido Hospital.

\section{METODOLOGIA}

Trata-se de um survery descritivo e exploratório ${ }^{(13)}$ que

coletam descrições detalhadas de variáveis existentes e usam dados para justificar e avaliar condições de práticas correntes ou fazer planos mais inteligentes para melhorar as práticas de atenção à saúde.

O estudo ocorreu em um Hospital Público Estadual de Ensino do Município de São Paulo.

A amostra constitui-se de 100 mulheres internadas para cirurgias ginecológicas e que se encontravam entre o primeiro e o quinto dia de pós-operatório. Para facilitar a obtenção de informações mais precisas e consequientemente, um raciocínio clínico adequado, utilizou-se como critérios de inclusão, mulheres que não apresentavam queixas álgicas importantes, que demonstrassem boa comunicação e disposição no momento da coleta de dados e que não estivessem com acompanhante no momento da realização da entrevista e exame físico, após terem concordado livremente em participar do estudo.

$\mathrm{O}$ instrumento de coleta de dados, resultado principal deste estudo, foi elaborado segundo os Padrões Funcio- 
nais de Saúde ${ }^{(14)}$, citados adiante, e com base no Modelo Bifocal da Prática Clínica ${ }^{(6)}$, apresentado em 1983. Segundo esta autora,

representa as situações que influenciam as pessoas, os grupos e as comunidades, assim como a classificação das suas respostas pela perspectiva de enfermagem.

Estas situações são organizadas em cinco amplas categorias: fisiopatológicas; relacionadas com o tratamento; pessoais; ambientais e maturacionais. O instrumento contém dados sócio-demográficos, variáveis gineco/obstétricas, e de hábitos de vida e diagnósticos identificados.

A coleta de dados foi realizada no período de maio a setembro de 2002, após aprovação pelo Comitê de Ética em Pesquisa da UNIFESP/EPM e do Hospital do Mandaqui e o consentimento livre e esclarecido das participantes do estudo.

\section{RESULTADOS}

Ressalta-se que a maioria das mulheres que participou do estudo tinha idade entre 28 a 57 anos $(60,0 \%)$, era de cor branca $(54,0 \%)$, tinha como ocupação atividades do lar $(55,0 \%)$. A maioria encontrava-se entre o primeiro e segundo dia de pós-operatorio e as cirurgias mais freqüentes foram: histerectomia abdominal por leiomiomas $(31,0 \%)$, Colpoperinieoplastia por prolapso genital (13,0\%), Curetagens de prova, e conização $(9,0 \%)$ respectivamente e mastectomia por câncer de mama em $8,0 \%$.

O instrumento construído, caracterizou o objetivo da pesquisa, após várias aplicações em pré-testes até chegar-se a este modelo. É composto de Anamnese e Exame Fisico, contendo na sua estrutura, dados de identificação; sócios demográficos; variáveis gineco/obstétricas; variáveis de hábitos de vida; patologias e cirurgias realizadas, distribuídos de acordo com os Padrões Funcionais de Saúde ${ }^{(14)}$, relacionados a seguir:

\section{Valor - Crença}

Descreve os padrões de valores, objetivos e crenças (incluindo espirituais) que guiam as escolhas e decisões. Inclui o que é percebido como importante na vida e quaisquer conflitos detectados em valores, crenças ou expectativas relacionadas à saúde.

\section{Percepção de Saúde - Controle de Saúde}

Descreve o padrão de saúde e bem-estar percebido do cliente e como sua saúde é controlada. Inclui a per- cepção do cliente do seu próprio status de saúde e sua relevância para atividades correntes e planejamentos futuros. Também inclui o nível geral de comportamento de cuidados de saúde, tais como atividades de promoção de saúde, participação em práticas preventivas de saúde mentais e físicas, prescrições médicas ou de enfermagem e cuidados de acompanhamento.

\section{Sexual - Reprodutivo}

Descreve o padrão de satisfação ou insatisfação com a sexualidade e o padrão reprodutivo. Inclui a satisfação percebida ou distúrbios na sexualidade ou em relações sexuais. Também estão incluídos o estado reprodutivo feminino, pré-menopausa ou pós-menopausa e quaisquer problemas detectados.

\section{Papel - Relacionamento}

Descreve o padrão de papel em compromissos e relacionamentos. Inclui a percepção dos principais papéis e responsabilidades na situação corrente da vida do cliente. São incluídos satisfações ou distúrbios na família, trabalho ou relações sociais, bem como as responsabilidades relacionadas a estes papéis.

\section{Cognitivo - perceptivo}

Descreve o padrão cognitivo e de percepção dos sentidos. Inclui a adequação dos modos sensoriais, como visão, audição, paladar, tato e olfato, e a compensação ou próteses utilizadas para tratamentos de distúrbios. Quando for o caso, são incluídos relatórios de percepção de dor e de como esta é gerenciada. São descritas habilidades funcionais cognitivas, como linguagem, memória, julgamento e tomada de decisão.

\section{Atividade - Exercícios}

Descreve o padrão de atividades relacionadas a exercícios, lazer e recreação. Inclui atividades do dia-a-dia que requerem gasto de energia, como manter a higiene, cozinhar, fazer compras, comer, trabalhar ou cuidar da casa. Também são incluídos tipo, quantidade e qualidade do exercício, inclusive exportes, que descrevem o padrão típico. (São incluídos fatores que interferem no padrão desejado ou esperado para um indivíduo, tais como déficits e compensações neuromusculares, dispnéia, angina ou cãibras musculares quando de esforços, e, se for o caso, classificação cardíaca e pulmonar). Padrões de lazer são incluídos e descrevem atividades de recreação realizadas com ou sem outros indivíduos. A ênfase deve ser nas atividades de maior importância para o cliente. 


\section{Sono - Repouso}

Descreve os padrões de sono, descanso e relaxamento, incluindo os períodos de cada um destes durante um dia completo. Inclui a percepção de qualidade e quantidade de sono e descanso e a percepção de nível de energia. Também são incluídos dispositivos de auxílio ao sono, como medicamentos ou rotinas noturnas.

\section{Autopercepção - Autoconceito}

Descreve o padrão de auto-conceituação e da percepção de si mesmo. Inclui atitudes do indivíduo com relação a si próprio, percepção de habilidades (cognitivas, afetivas e físicas), imagem, identidade, senso geral de valor, e padrão emocional geral. Também são incluídos padrões de postura do corpo e movimentos, contato visual e padrões de voz e de discurso.

\section{Enfrentamento- Tolerância ao estresse}

Descreve o padrão geral de superação e sua efetividade em termos de tolerância ao stress. Inclui a reserva ou capacidade de resistir a desafios à integridade pessoal, modos de gerenciamento de stress, família ou outros sistemas auxiliares, e a habilidade percebida de controlar e gerenciar situações.

\section{Eliminatório}

Descreve padrões das funções excretoras (intestino, bexiga e pele) dos indivíduos. Inclui a percepção pelo indivíduo da regularidade das funções excretoras, uso de rotinas ou laxantes para limpeza do intestino e quaisquer mudanças ou distúrbios em padrões de tempo, modo de excreção, qualidade e quantidade. Também são incluídos quaisquer dispositivos empregados para controlar a excreção e, quando apropriado, o padrão de coleta de dejetos feita pela família ou comunidade.

\section{Nutricional - Metabólico}

Descreve padrões de consumo de alimentos e líquidos com relação às necessidades metabólicas e indicadores de padrão de suprimentos locais de nutrientes. Inclui os padrões de consumo de alimentos e líquidos do indivíduo, horários diários de alimentação, tipos e quantidades de alimentos e líquidos consumidos, preferências particulares com relação a certos alimentos e uso de nutrientes ou vitaminas suplementares. Também são incluídos relatórios sobre quaisquer lesões na pele e capacidade geral de regeneração e ainda, as condições da pele, cabelo, unhas, membranas mucosas e dentes, bem como medidas da temperatura do corpo, altura e peso e estado nutricional, como peso e ingesta alimentar.

Apresenta-se a seguir, o modelo definitivo do instrumento de coleta de dados para a sistematização da assistência de enfermagem ginecológica ${ }^{(15)}$.

\section{INSTRUMENTO DE COLETA DE DADOS PARA A IDENTIFICACÃ̃O DOS DIAGNOSTICOS DE ENFERMAGEM EM CLÍNICA GINECOLÖGICA}

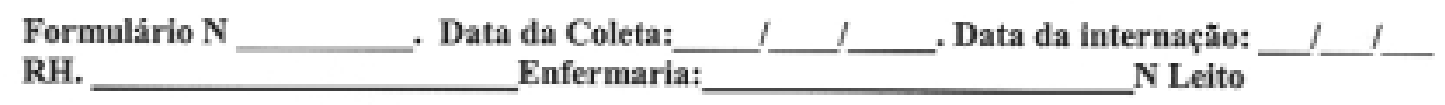

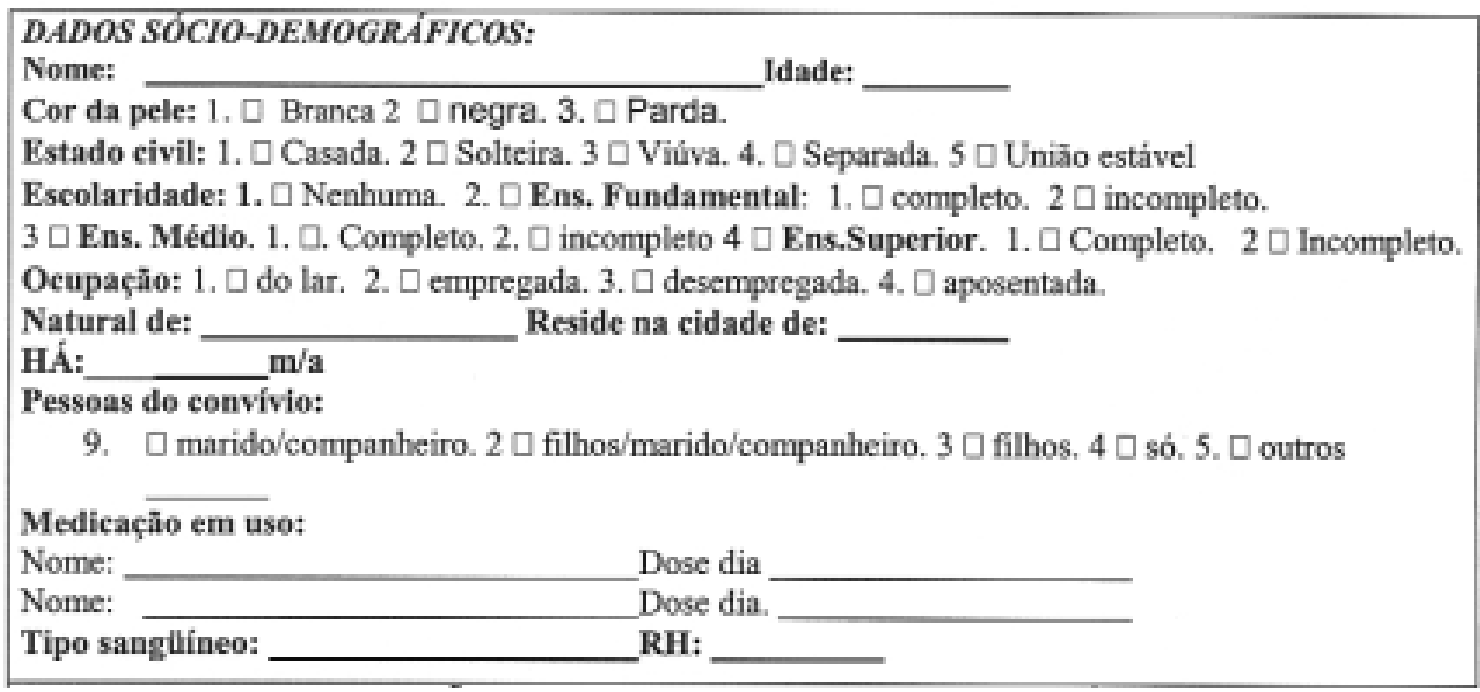


continuação...

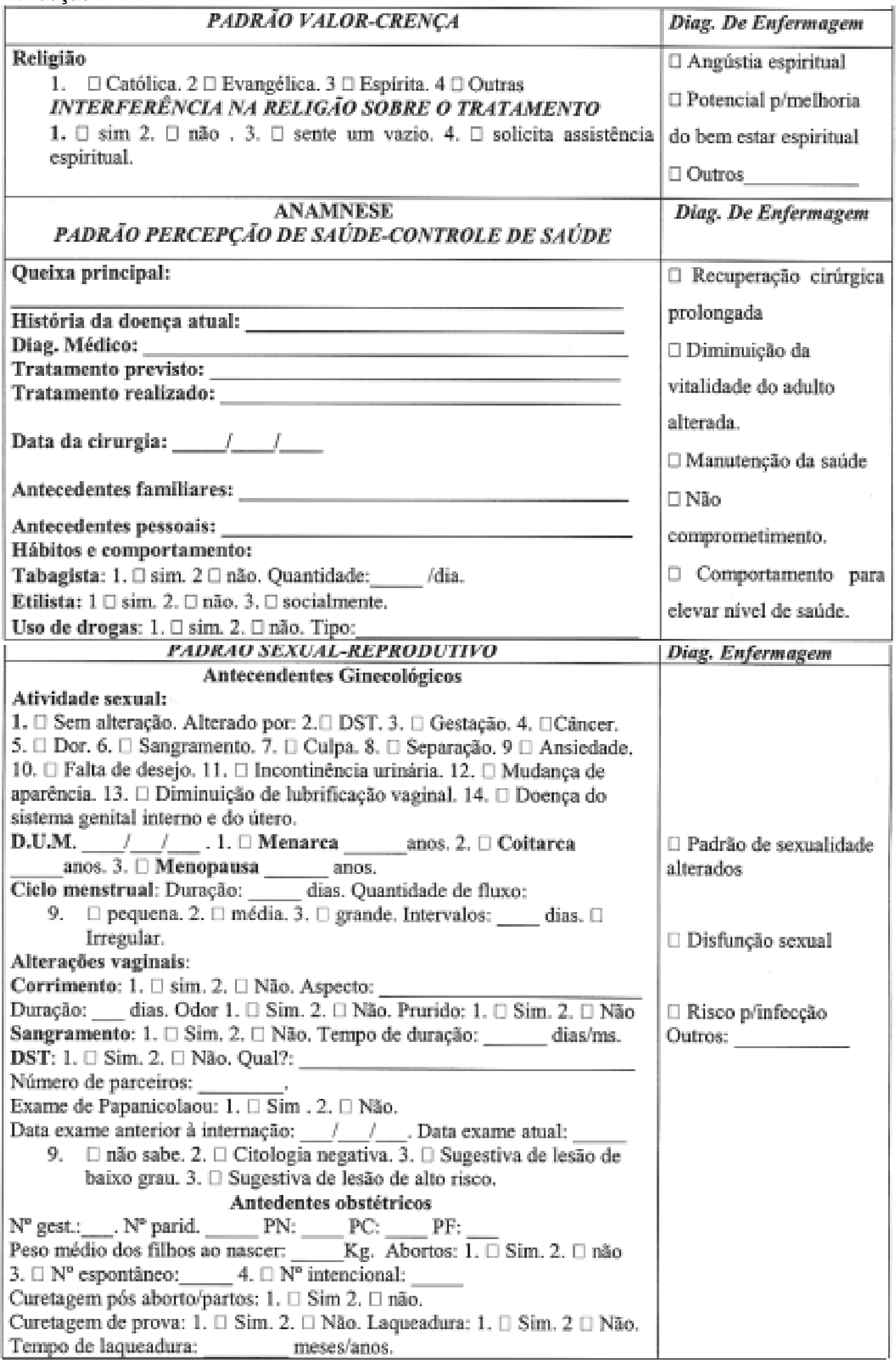


continuação...

PADRAO: PAPEL-RELACIONAMENTO

A doença a afastou de: Trabalho: 1. $\square$ Sim. 2. Năo. Laser: 1. $\square$ Sim.

2. \ Não. Familiares: 1. D Sim. 2, $\square$ Não.

Interação Social:

1. $\square$ Normal. Prejudicada por: 2. $\square$ perda de parte do corpo. 3. $\square$ doença crỏnica, 4, $\square$ imaturidade emocional. $5 \square$ Respostas agressivas.

6. $\square$ Ansiedade. 7. $\square$ Crença/religiăı. 8. $\square$ Comportamento depressivo.

9. $\square$ Sentimento de rejeiçĩio. 10, $\square$ doença terminal Outros:

Nivel de consciência PADRAO: COGNTTIVO-PERCEPTIVO

1. $\square$ Acordada. 2. $\square$ Lúcida. 3. $\square$ Comatosa. 4. $\square$ Torporosa.

5. $\square$ Confusa. 6. $\square$ Com falhas de memória.

Comunicação

1. $\square$ Normal. 2. $\square$ mimica. 3. $\square$ Escrita.

Prejudicada por: 1. $\square$ Dispnéia. 2. $\square$ Dor. 3. $\square$ Fadiga. 4. $\square$ Ansiedade.

5. $\square$ Analgesia. Outros:

Interação com o estado de saúde

1. $\square$ Demonstra bom conhecimento sobre o atual estado de saúde.

2. Demonstra pouco conhecimento sobre a doença e tratamento.

3. $\square$ Tem dificuldade de entendimento;

4. $\square$ Nada sabe sobre a doença e tratamento

5. $\square$ Solicita informaçốes.

6. Interpreta de maneira errada as informações recebidas.

7. $\square$ Não tem interesse em aprender

8. $\square$ Responde lentamente a pergunta

9. $\square$ Apresenta outras necessidades.

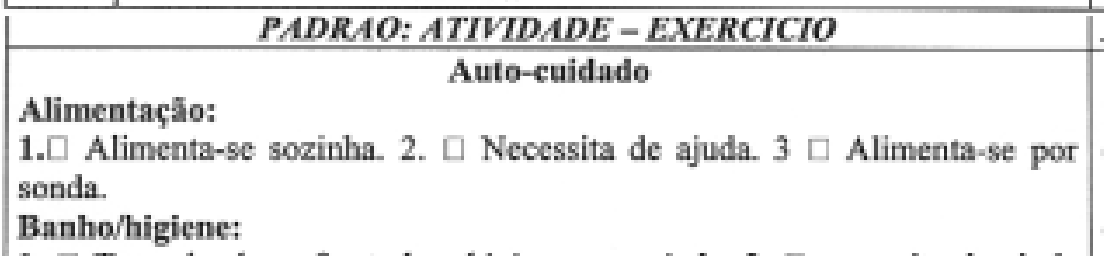

1. $\square$ Toma banho e faz toda a higiene sem ajuda. 2. $\square$ necessita de ajuda para banho/higiene.

Vestir-se/arrumar-se:

1. $\square$ Habilidade prejudicada para colocar e retirar a roupa.

2. $\square$ Não necessita de ajuda.

Oxigenação:

1. $\square$ ar ambiente. 2. $\square$ Oxigenoterapia. $3 \square$ Traqueostomia

Mobilidade:

1. $\square$ Deambula. 2. $\square$ Não deambula. $3 \square$ Deambula com ajuda. 4. $\square$ Dor pós cirurgia. 5. $\square$ Restrição imposta de movimentos. 6 . $\square$ Relutância em mover-se. 7. $\square$ Uso de prótese (muletas/andadaor). 8. $\square$ Movimenta-se no leito com ajuda.

\begin{tabular}{|l|l}
\hline PADRAOIO SONO-REPOUSO & DIE \\
\hline Sono: &
\end{tabular}

1. $\square$ Dorme bem. 2. $\square$ Dorme pouco. 3. $\square$ Insônia. 4. $\square$ Só dorme com medicação.

Dificuldade para adormecer:

1. $\square$ Hospitalização. $2 \square$ Acesso venoso. 3. $\square$ Dor. 4. $\square$ Preocupaçâo com familiares. Outros:

Repouso:

1. $\square$ Absoluto, 2. $\square$ Relativo. 3. $\square$ Sem restriç̄o.

Diag. Enfermagem

$\square$ Déficit no auto-

cuidado: banho/higiene.

$\square$ Déficit no auto-

cuidado:

vestir-se/arrumar-se.

$\square$ Mobilidade fisica

prejudicada.

प Intolerância à

atividade.

$\square$ Risco para função

respiratória alterada.

口 Perfusão tissular

periférica alterada.

$\checkmark$ Risco p/traumas.

Outros:

Diag. Enfermagem

प Distúrbio no padrão do sono.

૫ Privação do sono.

Outros: 
continuação...

\begin{tabular}{|c|c|}
\hline PADRAOAO: AUTOPERCEPCÄO-AUTOCONCEITO & Diag, Enfermagem \\
\hline $\begin{array}{l}\text { Sentimentos expressos sobre si: } \\
\text { 1. } \square \text { Preocupaçio. 2. } \square \text { Raiva. 3. } \square \text { ressentimento. 4. } \square \text { Inquietaçăo. } \\
\text { 5. } \square \text { Medo a respeito do significado da vida. 6. } \square \text { Medo do sofrimento. } \\
\text { 7. } \square \text { Medo da morte. Outros: } \\
\text { Atitudes expressas sobre si: } \\
\text { 1. } \square \text { Verbalização negativa sobre si. 2. } \square \text { Falta de confiança. 3. } \square \text { Recusa a } \\
\text { olhar-se no espelho, 4. } \square \text { Recusa-se a participar ou responsabilizar-se pelo } \\
\text { próprio cuidado. } 5 \text {. } \square \text { Má apresentaçăo corporal (postura, contato visual, } \\
\text { movimento), 6. } \square \text { Choro. Outros: }\end{array}$ & $\begin{array}{l}\square \text { Medo } \\
\square \text { Ansiedade } \\
\square \text { Baixa auto-estima } \\
\text { situacional } \\
\square \text { Baixa auto-estima } \\
\text { crônica. } \\
\square \text { Distúrbio na imagem } \\
\text { corporal } \\
\square \text { Distúrbio no } \\
\text { autoconceito } \\
\square \text { Impotência } \\
\square \text { Ansiedade relacionada } \\
\text { a morte } \\
\square \text { Desesperança. } \\
\text { Outros: }\end{array}$ \\
\hline$P A D R A$ & Diag, Enfermagem \\
\hline $\begin{array}{l}\text { Sentimentos e atitudes em relaçã̃o à doença: } \\
\text { 1. } \square \text { Descrença sobre o tratamento. 2. } \square \text { Dificuldade para tornar-se } \\
\text { independente. 3. } \square \text { Ausência de pensamento para o futuro. } 4 \square \\
\text { Verbalização aumentada das necessidades. 5. } \square \text { Verbalização diminuida } \\
\text { das necessidades. 6. } \square \text { Recusa em aceitar os esforços de reabilitaçăo. }\end{array}$ & $\begin{array}{l}\square \text { Enfrentamento } \\
\text { ineficaz } \\
\square \text { Adaptação prejudicada } \\
\square \text { Outros: }\end{array}$ \\
\hline $\begin{array}{l}\text { Eliminações urinárias: } \\
\text { 1. } \square \text { espontânea. 2. } \square \text { Polaciúria. 3. } \square \text { Oligúr } \\
\text { Incontinẻ̉ncia. 6. } \square \text { Irrigação, 7, } \square \text { SVD, 8. } \square \text { Disú } \\
\text { Outros: }\end{array}$ & $\begin{array}{l}\square \text { Risco para } \\
\text { constipação } \\
\square \text { Constipação } \\
\text { percebida } \\
\square \text { Constipação } \\
\square \text { Diarréia } \\
\square \text { Padrões de } \\
\text { eliminação urinária } \\
\text { alterada. } \\
\square \text { Incontinẻncia urinária } \\
\text { por pressão. } \\
\square \text { Outros: } \\
\end{array}$ \\
\hline \multicolumn{2}{|l|}{ EXAME FISICO } \\
\hline 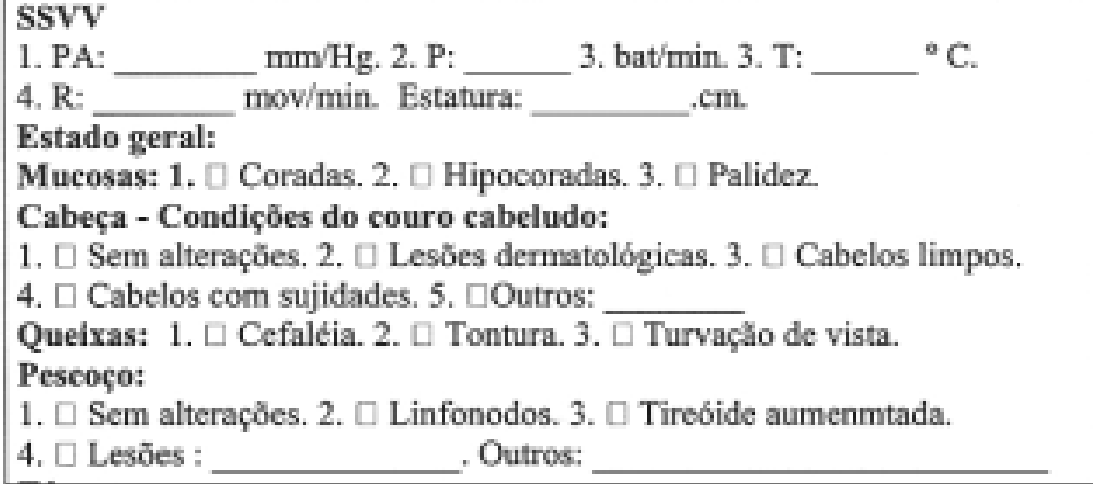 & $\square$ Hipotermia \\
\hline
\end{tabular}


continuação...

Tórax:

1. $\square$ Sem alteraçōes. 2. $\square$ Expansão normal. 3. $\square$ Expansằo diminuida. Ausculta pulmonar: 1. $\square$ normal. 2. $\square$ Alterada. 3. $\square$ Roncos. 4. $\square$

Estertores. 5. $\square$ Sibilos.

Coração: 1. $\square$ ritmico. 2. Taquicardia. 3. $\square$ Bradicardia. 4. $\square$ sopros. Mamas: 1. $\square$ Sem alteraçōes. 2. $\square$ Tumor fixo D/E 3. $\square$ Tumor móvel D/E 4. $\square$ Cicatriz cirúrgica anterior D/E. 5. $\square$ Nodulo em regiăo axilar D/E. 6. $\square$ Presença secreçào mamilo $\mathrm{D} / \mathrm{E}$. 7. $\square$ Lesōes ulcerativas D/E.

8. D Secreçã̃o em lesăo D/E. 9. D Outras alteraçỏes:

Membros superiores: 1. $\square$ Sem alteraçōes: 2. $\square$ Acesso venoso D/E.

3. $\square$ Linfedema D/E. 4. Edema de braço D/E. 5. $\square$ Edema de mão D/E.

6. $\square$ Rigidez de dedos da mão D/E. 7. $\square$ Diminuiçăo da sensibilidade $\mathrm{D} / \mathrm{E}$.

8. $\square$ Outros:

Abdome: 1. $\square$ Plano, 2, 0 Flácido. 3. $\square$ Distendido. 4. $\square$ Indolor.

5. $\square$ Doloroso. 6. $\square$ Presença de tumoração. $7 \square$ Ruidos H.A. presentes.

8. $\square$ Ruidos H.A. diminuidos. 9. $\square$ Ruidos H.A. ausentes.

Incisão círúrgica: $1 \square$ Recente limpa e seca. 2. $\square$ Processo inflamatório.

3. $\square$ Deiscência parcial. 4. $\square$ Deiscência total. 5 , $\square$ Secreção:

caracteristica:

Genitália externa: 1. $\square$ Sem alteraçōes; 2. ㅁ Pilificação normal.

3. $\square$ Pilificação alterada. 4. $\square$ anomalia:

4. $\square$ Lesỗes tipo:

Membros inferiores: 1. $\square$ Sensibilidade e força motora preservada D/E.

2. $\square$ Cianose de extremidades D/E. 3. $\square$ Varizes D/E. 4. Edema D/E.

Lesōes D/E tipo:

Enfermeira/o.

. COREN:

$\square$ Risco para

transmissão de infeção

$\square$ Déficit de volume de liquido.

$\square$ Excesso de volume de liquido.

$\square$ Integridade da pele prejudicada

○ Proteçã̃o alterada

Outros:
A partir da aplicação deste instrumento foi possível identificar 48 diagnósticos de enfermagem, dentre eles, oito diagnósticos com freqüência entre $70,0 \%$ a $100,0 \%$, nove diagnósticos entre $40,0 \%$ a $69,0 \%$ e os demais, com $31,0 \%$ a $64,0 \%$. Apresenta-se abaixo, os dois primeiros grupos de diagnósticos.

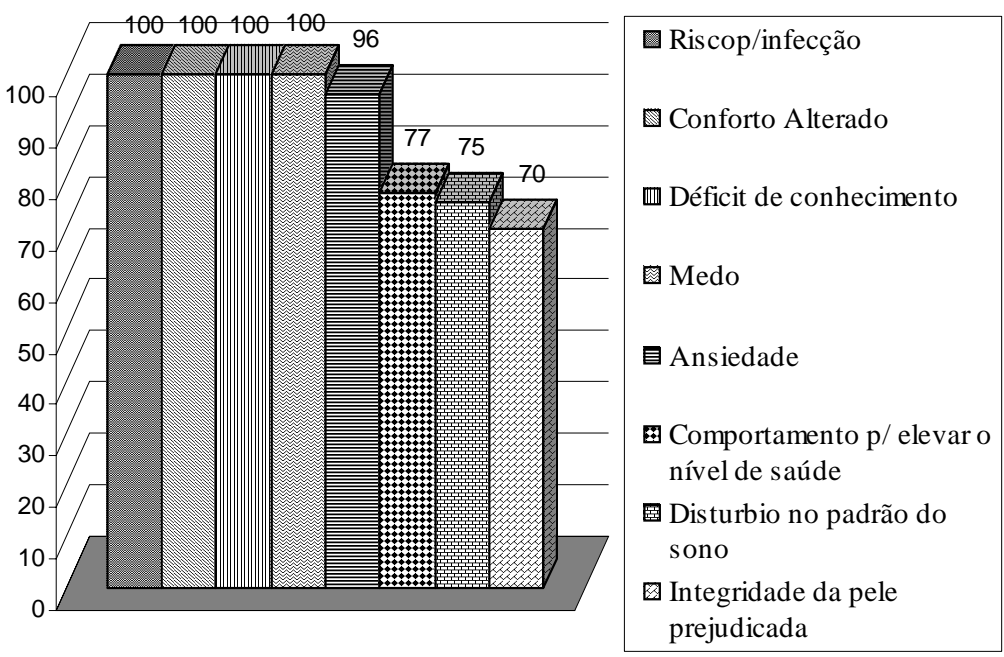

Figura 1 - Diagnósticos de enfermagem identificados com freqüência de 70,0\% a 100,0\% em mulheres atendidas em um Hospital Público Estadual de Ensino no Município de São Paulo. São Paulo, 2002. 


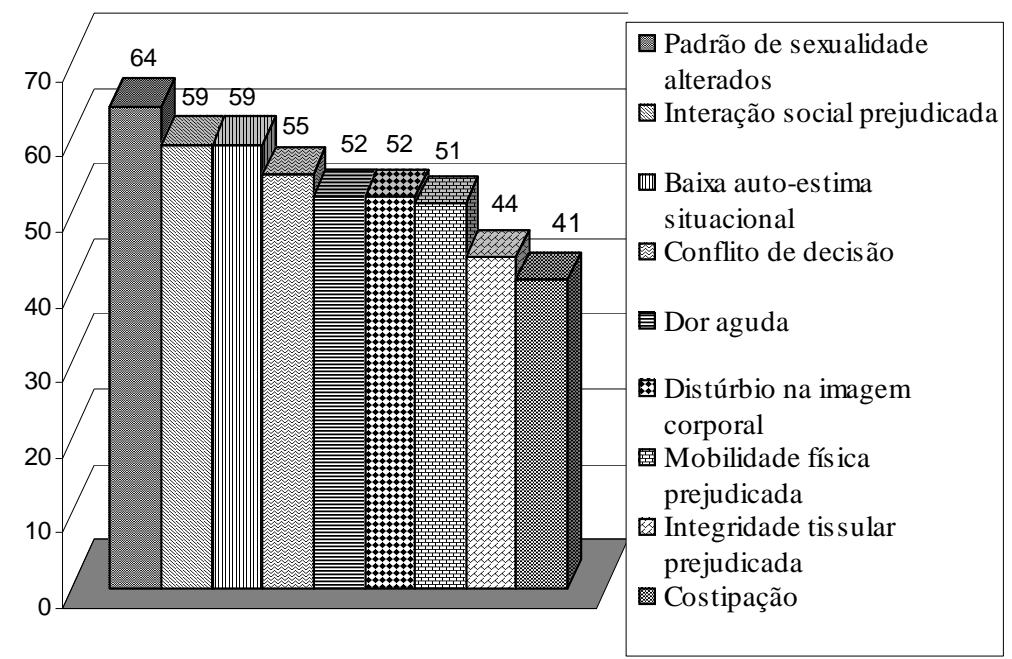

Figura 2 - Diagnósticos de enfermagem identificados com freqüência de 40,0\% a 69,0\% em mulheres atendidas em um Hospital Público Estadual de Ensino no Município de São Paulo. São Paulo, 2002.

\section{CONCLUSÃO E CONSIDERAÇÕES FINAIS}

O instrumento mostrou-se adequado para a coleta de dados em clínica cirúrgica de ginecologia, uma vez que permitiu a identificação de 17 diagnósticos de enfermagem com frequiência de $40,0 \%$ a $100,0 \%$ na clínica em estudo. Os resultados obtidos favorecem a sistematização da assistência de enfermagem, gerando ações individualizadas, e avaliação durante a internação das pacientes.

Considera-se o estudo fundamental para o desenvolvimento da prática clínica de enfermagem, uma vez que o instrumento possibilita a informatização da prática de enfermagem e melhor qualidade de assistência.

\section{REFERÊNCIAS}

1. Alfaro-Lefevre R. Aplicação do processo de enfermagem: um guia passo a passo. Trad. de Ana Maria Thorell. $4^{\mathrm{a}}$ ed. Porto Alegre: Artmed; 2000. Investigação; p. 52-89.

2. Cianciarullo TI. Instrumento básico para o cuidar: um desafio para a qualidade de assistência. São Paulo: Atheneu; 2000. Instrumentos básicos: como usá-los na enfermagem.; p. 1-4.

3. Farias NJ, Nóbrega LMM, Pérez BLV, Coler SM. Diagnóstico de enfermagem: uma abordagem conceitual e prática. Paraíba: Reprint; 1990. Histórico do diagnóstico de enfermagem; p. 23-43.

4. North American Nursing Association (NANDA). Nursing diagnosis: definitions \& classification 2001-2002. Philadelphia; 2002.

5. Doenges MF, Moorhouse MF. Diagnóstico e intervenção em

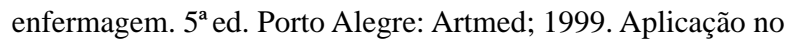
processo de enfermagem; p. 18-21.

6. Carpenito LJ. Diagnósticos de enfermagem: aplicação à prática clínica. $8^{\mathrm{a}}$ ed. Trad. de Ana Maria Thorell. Porto Alegre: Artmed; 2002.
7. Horta WA. Processo de enfermagem. São Paulo: EPU; 1979. p. 35-74.

8. Gutierrez BAO, Soares AVN, Anabuki MH, Nomura FH. Histórico de enfermagem. In: Cianciarullo TI, Gualda DMR, Melleiro MM, Anabuki MH. Sistema de assistência de enfermagem: evolução e tendências. São Paulo: Ícone; 2002. p.131-64

9. Abrão ACFV. Diagnóstico de enfermagem em aleitamento materno: estudo de validação. [tese doutorado]. São Paulo: Universidade Federal de São Paulo; 1998.

10. Lacava RMVB. Elaboração e aplicação de um instrumento de levantamento de dados e diagnósticos de enfermagem em gestantes. [tese doutorado] .São Paulo: Universidade Federal de São Paulo; 1999.

11. Vieira ES. Identificação e validação dos diagnósticos de enfermagem" Padrões de sexualidade alterados e disfunção sexual". [tese doutorado]. São Paulo: Universidade Federal de São Paulo; 2001.

12. Gerk MAS. Saúde da mulher: conjunto prioritário de diagnóstico de enfermagem em ginecologia. [tese doutorado]. São Paulo: Universidade Federal de São Paulo; 1999.

13. Lobiondo-Wood G, Haber J. Desenhos não-experimentais. $4^{\text {a }}$ ed. Trad. de Ivone Evangelista Cabral. Rio de Janeiro: Guanabara Koogan; 2001. O papel da pesquisa em enfermagem; p. 111-21

14. Gordon M. Nursing diagnosis: process and aplications. St. Louis: Mosby; 1994.

15. Soares LH. Construção de um instrumento de coleta de dados para a identificação dos diagnósticos de enfermagem em ginecologia. [tese doutorado]. São Paulo: Universidade Federal de São Paulo; 2003. 